\section{Liquid Drops on the Same Liquid Surface.}

Dr. M. Katalinić has described a method of producing floating drops of clean water on a water surface by delivery from a suitably situated jet. ${ }^{1}$ Such drops must owe their existence to the stability of the film immediately beneath them, that is, the thicknesscoefficient of the surface tension of the film over a suitable range must be negative, a characteristic of all stable films such as those of soap. Therefore a drop of soap solution should be stable on a water surface, the hydrophobic ends of the soap ions in the surface being directed outwards, and we were able last August to produce such drops of any diameter up to about $1 \mathrm{~cm}$. by simply dipping a piece of soap (any kind) in water and allowing the drops to fall off it on to the surface from a height of about $1 \mathrm{~cm}$. It is best to draw the soap down in a sweeping curve into the water and then out. In this way the floating drops are propelled forward and travel for distances up to quite 1 metre, if they are not too large. The very large drops last for scarcely one second, but the smaller ones for much longer. We experimented in a large tank of rain water illuminated by the sun, and interference colours were easily observed in the film beneath the drops. Later we obtained the same results in a beaker of distilled water, or even in tap water. The observations we made were, in the main, the same as those recorded by Dr. Katalinić.

Our main object in this communication, however, is to record an observation which to us is new, namely, that in addition to the floating drops, there are often produced, but in much smaller numbers, drops of about $1.4 \mathrm{~mm}$. diameter which sink to a considerable depth and slowly rise again, coming to rest immediately underneath the surface. The soap film surrounding these drops is evident by the interference colours observed. They are stable for at least a minute, although they immediately burst on being touched, for example, with a clean platinum wire. They occasionally contain a minute air bubble of about $0 \cdot 1 \mathrm{~mm}$. diameter.

We are of the opinion that these submerged drops are formed by the drop of soap solution sinking into and getting encased entirely in the main bulk of the water, because occasionally, on slowly arriving upwards at the surface, they break through and eject their own contents some millimetres above the surface, in the form of a drop which bounces several times on the surface before coming to rest and ultimately coalescing. We recently found that these submerged drops are easiest obtained in tap water.

William Hughes.

King Edward VI. School, Southampton, Dec. 11 .

1 Nature, 127, 627, April 25, 1931.

\section{A New Method of Investigating the Modes of Vibration of Quartz Crystals.}

A NEW method of investigating the modes of vibra. tion of quartz crystals has arisen out of the use of E. P. Tawil's 1 development of Töpler's Schlieren method. Tawil was able, by utilising variations of refractive index, to photograph stationary or progressive supersonic waves in air.

In this experiment, if an accurately worked quartz crystal is set up in the incident light-beam, it is possible to orientate the crystal so that the light transmitted through it is undeviated; consequently, if the crystal is not oscillating, the whole of the field will appear dark when viewed through the telescope.
As soon as the crystal is set oscillating it will be found that certain parts of the crystal will light up, leaving in most cases a pattern of dark bands. If a fundamental mode is used, the appearance of the system will be as shown in Fig. 1, A; if a more com. plex vibration is obtained, a correspondingly complex pattern results, as shown in Fig. 1, B.

It is clear that those parts remaining dark are not giving any deviation of the incident light, and consequently represent nodal lines. However, the interpretation of the results will be complicated by the

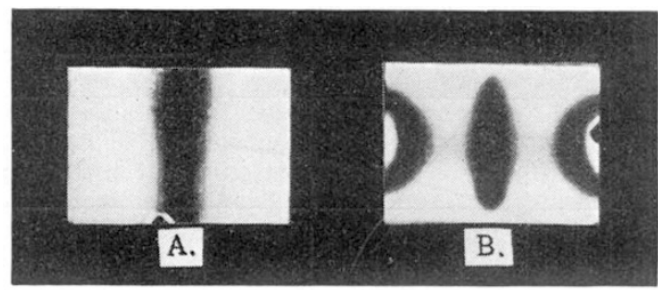

Frg. 1.

fact that the pattern is probably the mean result of all figures in all planes perpendicular to the light-beam.

The method appears to possess these advantages :

(1) The effect is easily obtained even with low intensities of oscillation.

(2) The result is readily photographed.

(3) The effect is one of black-and-white contrast and consequently stands out well in cases where a coloured field might confuse the result.

In short, it seems that this additional method of examining the modes of vibration of quartz plates will, when combined with a polarised light method, give very useful results.

Physics Department,

Victoria University College,

Wellington, New Zealand, Nov. 3.

1 Comptes rendus, 191, pr. $998-1000 ; 1930$.

\section{An Electrostatic Explanation of the Phenomenon of Flotation.}

THE explanation of the flotation processes with the aid of the cataphoretic and electroendosmotic potentials has failed completely. These potentials play an important part at a distance from the geometrical interface of the phase only. ${ }^{1}$ There are, however, suggestions in the literature ${ }^{2}$ that ideal chemical electrodes should have a positive electrostatic poten. tial in water and the dielectrics a negative one. This suggestion has important consequences. Emulsions of dielectrics in water will wet unattackable electrodes immersed in water but not attackable electrodes which are coated with dielectric oxides (hydroxides) in water. The wetting must, however, reduce the positive charge of the unattackable electrode, and it is expected that the wetting will not take place on attackable electrodes coated with a dielectric hydroxide, as for example, zinc. This is actually observed.

The irreversible electrostatic potential on the interface solid-liquid was measured with the aid of a Lindemann, or Leiss Perucca electrometer of small capacity. A platinum electrode, a galena electrode, an Acheson graphite electrode for spectroscopic pur. poses made by Messrs. Adam Hilger, Ltd., and a zinc electrode, were immersed successively in 50 c.c. of $0.002 \mathrm{~N}$ potassium chloride of $p \mathrm{H} 7.5$ and their potential was measured against a $0.002 N$ calomel

No. 3245, VoL. 129] 